\title{
A LIOUVILLE THEOREM FOR THE COMPLEX MONGE-AMPÈRE EQUATION ON PRODUCT MANIFOLDS
}

\author{
HANS-JOACHIM HEIN
}

\begin{abstract}
Let $Y$ be a closed Calabi-Yau manifold. Let $\omega$ be the Kähler form of a Ricci-flat Kähler metric on $\mathbb{C}^{m} \times Y$. We prove that if $\omega$ is uniformly bounded above and below by constant multiples of $\omega_{\mathbb{C}^{m}}+\omega_{Y}$, where $\omega_{\mathbb{C}^{m}}$ is the standard flat Kähler form on $\mathbb{C}^{m}$ and $\omega_{Y}$ is any Kähler form on $Y$, then $\omega$ is actually equal to a product Kähler form, up to a certain automorphism of $\mathbb{C}^{m} \times Y$.
\end{abstract}

\section{INTRODUCTION}

The purpose of this article is to prove the following theorem.

Theorem A. Let $Y$ be a closed Kähler manifold with $c_{1}(Y)=0$ in $H^{2}(Y, \mathbb{R})$. Let $\omega$ be a Ricci-flat Kähler form on $\mathbb{C}^{m} \times Y$. Assume that $C^{-1}\left(\omega_{\mathbb{C}^{m}}+\omega_{Y}\right) \leqslant \omega \leqslant C\left(\omega_{\mathbb{C}^{m}}+\omega_{Y}\right)$ for some $C \geqslant 1$, where $\omega_{\mathbb{C}^{m}}$ is the standard flat Kähler form on $\mathbb{C}^{m}$ and $\omega_{Y}$ is any Kähler form on $Y$. Then:

(1) There is a unique choice of $\omega_{Y}$ such that $\operatorname{Ric}\left(\omega_{Y}\right)=0$ and $\omega$ is d-cohomologous to $\omega_{\mathbb{C}^{m}}+\omega_{Y}$. Fix this choice of $\omega_{Y}$. Then $\omega^{m+n}$ is a constant multiple of $\omega_{\mathbb{C}^{m}}^{m} \wedge \omega_{Y}^{n}$, where $n=\operatorname{dim}_{\mathbb{C}} Y$.

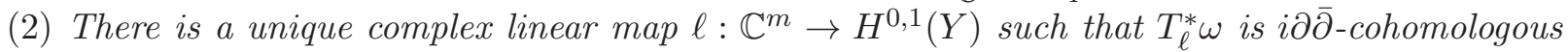
to $\omega_{\mathbb{C}^{m}}+\omega_{Y}$, where $T_{\ell}$ denotes the automorphism of $\mathbb{C}^{m} \times Y$ defined in (1.1) below.

(3) There is a matrix $S \in \mathrm{GL}(m, \mathbb{C})$ such that $T_{\ell}^{*} \omega=S^{*} \omega_{\mathbb{C}^{m}}+\omega_{Y}$.

Here (1) is obvious from Yau's theorem [19] and the fact that $\log \left(\omega^{m+n} / \omega_{\mathbb{C}^{m}}^{m} \wedge \omega_{Y}^{n}\right)$ is a bounded pluriharmonic function on $\mathbb{C}^{m} \times Y$, hence on $\mathbb{C}^{m}$, if $\operatorname{Ric}\left(\omega_{Y}\right)=0$. For $(2)$, we identify $H^{0,1}(Y)$ with the space of parallel $(0,1)$-forms with respect to the Ricci-flat metric $\omega_{Y}$ [1, Thm 6.11]. Then for all $\xi \in H^{0,1}(Y)$ we have a unique $\omega_{Y}$-parallel $(1,0)$-vector field $\xi^{\sharp}$ with $\left.\xi^{\sharp}\right\lrcorner \omega_{Y}=\xi$. The map $\xi \mapsto \xi^{\sharp}$ is complex linear. Formally write the time-1 map of $\xi^{\sharp}$ on $Y$ as $y \mapsto y+\xi^{\sharp}$ and define

$$
T_{\ell}(z, y)=\left(z, y+\ell(z)^{\sharp}\right) .
$$

Thus, (2) is trivial if $Y$ is a point. In this case, (3) reduces to a classical Liouville theorem for the complex Monge-Ampère equation $\operatorname{det}\left(\phi_{z^{i} \bar{z}^{j}}\right)=1$ for a plurisubharmonic function $\phi: \mathbb{C}^{m} \rightarrow \mathbb{R}[14$. This Liouville theorem is a consequence of the Calabi-Yau $C^{3}$ estimate [3, 19. If $Y$ is not a point but is flat, then this classical method still shows that $\omega$ is flat, and (2) and (3) follow from this. If $Y$ is not flat, then this method breaks down due to the appearance of negative curvature terms in a Weitzenböck type computation. So one way to think of Theorem A is as a "vanishing theorem" for "nonlinear harmonic 2-forms" that cannot be proved using the Weitzenböck method.

It is a standard theme in analysis that conversely, Liouville theorems imply regularity theorems, e.g. see [4]. The special case of Theorem $₫$ where $\ell=0$ and $\left.\omega\right|_{\{z\} \times Y}=\omega_{Y}$ for all $z \in \mathbb{C}^{m}$ (a very strong assumption, which quickly reduces the claim to the classical case $n=0$ ) was used in [17] to establish higher regularity properties of collapsing Ricci-flat Kähler metrics and Kähler-Ricci flows on holomorphic fiber spaces with generic fiber $Y$. In [17], the assumption that $\left.\omega\right|_{\{z\} \times Y}=\omega_{Y}$ for all $z$ was justified by the deep technical results of [16]. It is thus worth noting that our work here does not rely on [16]. New applications of Theorem $\mathrm{A}$ to regularity problems will appear in [9].

Date: July 30, 2018.

Research partially supported by NSF grant DMS-1514709. 
Here is a sketch of our proof of Theorem $\AA$. For any constant vector field $\partial_{z}$ on $\mathbb{C}^{m}$, the function $\left|\partial_{z}\right|_{\omega}^{2}$ is $\omega$-subharmonic, and is constant if and only $\partial_{z}$ is $\omega$-parallel, thanks to the Bochner formula for the $\bar{\partial}$-operator on vector fields [1, Prop 4.79] and to the fact that $\operatorname{Ric}(\omega) \leqslant 0$. Motivated by [13, $\S 2]$, V. Tosatti and Y. Zhang (unpublished) observed that if $m=1$, then this idea suffices to prove Theorem $\mathrm{A}$ because a bounded subharmonic function on a parabolic manifold is constant.

To improve this strategy, we will first use the standard case $n=0$ of Theorem $\AA$ to prove that the tangent cone at infinity of $\left(\mathbb{C}^{m} \times Y, \omega\right)$ is $\left(\mathbb{C}^{m}, \omega_{\mathbb{C}^{m}}\right)$. Estimates for subharmonic functions then allow us to prove that $\left|\partial_{z}\right|_{\omega}^{2}$ is $\leqslant \frac{1}{2}$ globally (and is asymptotic to $\frac{1}{2}$ along almost every ray in $\mathbb{C}^{m}$ ). For $m>1$ this is still consistent with the behavior of nonconstant bounded subharmonic functions such as $\max \left\{0,1-|z|^{2-2 m}\right\}$. However, it turns out that essentially the same conclusions hold for the dual quantity $\sum_{j=1}^{m}\left|d z^{j}\right|_{\omega}^{2}=2 \operatorname{tr}_{\omega}\left(\omega_{\mathbb{C}^{m}}\right) \leqslant 2 m$, which is $\omega$-subharmonic because of the reverse inequality $\operatorname{Ric}(\omega) \geqslant 0$ [1, Ex 1.38]. (The details are more involved because the metric on the cotangent bundle diverges to infinity in the $Y$-directions when we blow down.) Then it suffices to note that

$$
m^{2}=\left(\sum_{j=1}^{m} d z^{j}\left(\partial_{z^{j}}\right)\right)^{2} \leqslant\left(\sum_{j=1}^{m}\left|d z^{j}\right|_{\omega} \cdot\left|\partial_{z^{j}}\right|_{\omega}\right)^{2} \leqslant\left(\sum_{j=1}^{m}\left|d z^{j}\right|_{\omega}^{2}\right)\left(\sum_{j=1}^{m}\left|\partial_{z^{j}}\right|_{\omega}^{2}\right) \leqslant m^{2} .
$$

This forces equality in our Bochner formulas, so that $\nabla^{\omega} \partial_{z^{j}}=0$ and $\nabla^{\omega} d z^{j}=0$ for all $j$. Moreover, $\left|d z^{j}\right|_{\omega}^{2}=\lambda\left|\partial_{z^{j}}\right|_{\omega}^{2}=\frac{\lambda}{2}$ for some $\lambda \geqslant 0$, and hence $\left|d z^{j}\right|_{\omega}^{2}=2$ because $\sum_{j=1}^{m}\left|d z^{j}\right|_{\omega}^{2}=2 m$.

The remainder of this paper is organized as follows. In Section 2 we give a more detailed outline of the proof of Theorem $\mathrm{A}$ as a sequence of six steps. Some of these steps are obvious or follow from the literature. We provide technical details for the remaining ones in Section 3 ,

I would like to thank Bianca Santoro and Valentino Tosatti for very useful discussions.

\section{Outline}

Step 1. Form the 1-parameter family $\omega_{t}=\Psi_{t}^{*}\left(e^{-t} \omega\right)$ of Ricci-flat Kähler metrics on $\mathbb{C}^{m} \times Y$, where $\Psi_{t}(z, y)=\left(e^{t / 2} z, y\right)$. As $t \rightarrow \infty$ this family connects $\left(\mathbb{C}^{m} \times Y, \omega\right)$ to its tangent cone at infinity. By assumption, $\omega_{t}$ is uniformly bounded above and below by fixed multiples of the Ricci-flat product metric $\omega_{\mathbb{C}^{m}}+e^{-t} \omega_{Y}$, and $\omega_{t}^{m+n}$ is equal to a fixed multiple of $e^{-n t} \omega_{\mathbb{C}^{m}}^{m} \wedge \omega_{Y}^{n}$.

Step 2. Use ideas from [15] to find a subsequence $\omega_{t_{i}}$ that converges in the $C_{l o c}^{1, \alpha}$ topology of Kähler potentials and in the weak topology of currents on $\mathbb{C}^{m} \times Y$ to $\operatorname{pr}_{\mathbb{C}^{m}}^{*}\left(\omega_{\infty}\right)$, where $\omega_{\infty}$ is a BedfordTaylor solution to a complex Monge-Ampère equation $\omega_{\infty}^{m}=c \omega_{\mathbb{C}^{m}}^{m}$ on $\mathbb{C}^{m}$ (here $c>0$ is a constant). Since our set-up differs slightly from the one of [15], some preliminary steps are required.

Step 2.1. Prove that $\omega$ differs from $\omega_{\mathbb{C}^{m}}+\omega_{Y}$ by $i \partial \bar{\partial}$ of some global Kähler potential on $\mathbb{C}^{m} \times Y$, after "translating" $\omega$ by some automorphism $T_{\ell}$ as in (1.1) if necessary (which does not affect the uniform equivalence of the two Kähler forms). In fact this is part of the content of Theorem $₫(2)$. From this step on, we will assume throughout the whole paper that $T_{\ell}=$ id.

Step 2.2. Prove that on $B_{1} \times Y$, where $B_{1}=B_{1}(0)$ is the standard unit ball in $\mathbb{C}^{m}$, the Ricci-flat metric $\omega_{t}$ differs from the model metric $\omega_{\mathbb{C}^{m}}+e^{-t} \omega_{Y}$ by $i \partial \bar{\partial} \phi_{t}$ with $\left|\phi_{t}\right| \leqslant C$.

Step 2.3. Use ideas from [15] to extract a sequence $\omega_{t_{i}}$ as described above. Thanks to Step 2]2, this is a direct adaptation (and simplification) of the proof of [15, Thm 4.1], so we omit the details. We only remark that unlike in [15, it is not obvious at this point that the limit $\omega_{\infty}$ will be independent of the sequence $t_{i}$, but for us it suffices to have convergence along one fixed sequence.

Step 3. By construction, the Bedford-Taylor solution $\omega_{\infty}$ to $\omega_{\infty}^{m}=c \omega_{\mathbb{C}^{m}}^{m}$ produced as a weak limit of a collapsing sequence $\omega_{t_{i}}$ in Step 2 satisfies $C^{-1} \omega_{\mathbb{C}^{m}} \leqslant \omega_{\infty} \leqslant C \omega_{\mathbb{C}^{m}}$ in the sense of currents on $\mathbb{C}^{m}$. By [18, Thm 1.7] (see [18, Prop 3.13] for the fact that the notions of a Bedford-Taylor and a 
viscosity solution are equivalent), whose proof made extensive use of Caffarelli's work as in [2], $\omega_{\infty}$ is then Hölder continuous and hence, by standard bootstrapping arguments, smooth and uniformly equivalent to $\omega_{\mathbb{C}^{m}}$. The standard Liouville theorem for the complex Monge-Ampère equation of [14] now tells us that $\omega_{\infty}=S^{*} \omega_{\mathbb{C}^{m}}$ for some matrix $S \in \mathrm{GL}(m, \mathbb{C})$. Thus, $\phi_{t_{i}} \rightarrow\left(S^{*}-\mathrm{id}^{*}\right)\left(|z|^{2} / 2\right)+\pi$ in $C_{\text {loc }}^{1, \alpha}\left(\mathbb{C}^{m} \times Y\right)$ for some pluriharmonic function $\pi$ on $\mathbb{C}^{m}$. Replacing $\omega_{t}$ by $\left(S^{-1}\right)^{*}\left(\omega_{t}\right)$ and $\phi_{t}$ by $\left(S^{-1}\right)^{*}\left(\phi_{t}-\pi\right)+\left(\left(S^{-1}\right)^{*}-\mathrm{id}^{*}\right)\left(|z|^{2} / 2\right)$, we may then assume throughout the rest of the paper that $S=\mathrm{id}$, and in fact $\omega_{t}=\left(\omega_{\mathbb{C}^{m}}+e^{-t} \omega_{Y}\right)+i \partial \bar{\partial} \phi_{t}$ with $\phi_{t_{i}} \rightarrow 0$ in $C_{l o c}^{1, \alpha}\left(\mathbb{C}^{m} \times Y\right)$.

Step 4. It follows trivially from Step 3 that for any constant holomorphic vector field $\partial_{z}$ on $\mathbb{C}^{m}$, the component function $\left|\partial_{z}\right|_{\omega_{t_{i}}}^{2}$ converges to the constant $\frac{1}{2}$ in the sense of distributions on $\mathbb{C}^{m} \times Y$. On the other hand, the dual quantity $|d z|_{\omega_{t_{i}}}^{2}$, while uniformly bounded, does not obviously converge to anything (even in the sense of distributions) because the metric on the cotangent bundle induced by $\omega_{t}$ diverges in the fiber directions. To fix this problem, we will prove that the sum $\sum_{j=1}^{m}\left|d z^{j}\right|_{\omega_{t_{i}}}^{2}$ converges to $2 m$ in the sense of distributions, provided that we restrict ourselves to test functions pulled back from the base. This strongly uses the convergence $\phi_{t_{i}} \rightarrow 0$ at the potential level.

Step 5. We now promote the distributional convergence $\left|\partial_{z}\right|_{\omega_{t_{i}}}^{2} \rightarrow \frac{1}{2}$ and $\sum_{j=1}^{m}\left|d z^{j}\right|_{\omega_{t_{i}}}^{2} \rightarrow 2 m$ of Step 4 to stronger forms of convergence, using the fact that both of these functions are $\omega_{t_{i}}$-subharmonic (which has not played any role so far). To this end, we first adapt a standard property of sequences of subharmonic functions on a ball in Euclidean space [10, Thm 3.2.12] to prove that:

Step 5.1. We have $\left|\partial_{z}\right|_{\omega_{t_{i}}}^{2} \rightarrow \frac{1}{2}$ and $\sum_{j=1}^{m}\left|d z^{j}\right|_{\omega_{t_{i}}}^{2} \rightarrow 2 m$ in $L_{l o c}^{1}\left(\mathbb{C}^{m} \times Y\right)$. This proof also relies directly on the fact that $\operatorname{Ric}\left(\omega_{t_{i}}\right) \geqslant 0$ via an application of Li-Yau type heat kernel estimates.

Using subharmonicity once again gives us pointwise bounds. This is similar to [10, Thm 3.2.13], but as in Step 51 we need to replace the Euclidean methods of [10] by a heat kernel estimate.

Step 5.2. We have $\sup _{B_{1} \times Y}\left|\partial_{z}\right|_{\omega_{t_{i}}}^{2} \leqslant \frac{1}{2}+o(1)$ and $\sup _{B_{1} \times Y} \sum_{j=1}^{m}\left|d z^{j}\right|_{\omega_{t_{i}}}^{2} \leqslant 2 m+o(1)$ as $i \rightarrow \infty$.

The second statement is similar to a trace estimate [16, Lemma 4.7] that played a crucial role in [16] in a different setting and was quite difficult to prove there (using different methods).

Step 6. Undoing the scaling and stretching of Step 1 , the result of Step [5 says that on $\left(\mathbb{C}^{m} \times Y, \omega\right)$, the subharmonic functions $\left|\partial_{z}\right|_{\omega}^{2}$ and $\sum_{j=1}^{m}\left|d z^{j}\right|_{\omega}^{2}$ are $\leqslant \frac{1}{2}$ and $\leqslant 2 m$ globally (and are asymptotic to these constants along almost every ray in $\mathbb{C}^{m}$ ). Combining this with (1.2), we deduce that they are constant equal to $\frac{1}{2}$ and $2 m$, respectively. The equality case in the Bochner formula now shows that $\partial_{z^{j}}$ and $d z^{j}$ are $\omega$-parallel for all $j$. It follows from a Riemannian argument, together with the fact that $\omega$ is $i \partial \bar{\partial}$-cohomologous to $\omega_{\mathbb{C}^{m}}+\omega_{Y}$, that $\omega=\omega_{\mathbb{C}^{m}}+\omega_{Y}$, proving Theorem $\mathbb{A}(3)$.

\section{Proofs}

Step 1 is self-explanatory, and details for Steps 2.3 and 3 are available from the literature. Thus, it remains to prove the claims made in Steps 2,1, 2,2, and 4, 5, 6. All notation and conventions of Sections 12 will remain in force throughout this section. Because of this, some results actually hold in slightly greater generality than stated. For example, in Proposition 3.1, the $\omega_{\mathbb{C}^{m}}$ term and the fact that $\operatorname{Ric}(\omega)=0$ play no role, but our standing assumptions that $\omega-\left(\omega_{\mathbb{C}^{m}}+\omega_{Y}\right)$ is $d$-exact (by the conventions of Theorem $\underline{A}(1))$ and that $\operatorname{Ric}\left(\omega_{Y}\right)=0$ are crucial (although the latter could be relaxed to the conclusion of the Beauville-Bogomolov-Calabi decomposition theorem).

3.1. Kähler potentials. The following propositions deal with Steps 2 , 1 and 2,2, respectively.

Proposition 3.1. There exists a unique complex linear map $\ell: \mathbb{C}^{m} \rightarrow H^{0,1}(Y)$ such that $T_{\ell}^{*} \omega$ is $i \partial \bar{\partial}$-cohomologous to $\omega_{\mathbb{C}^{m}}+\omega_{Y}$, where $T_{\ell}$ denotes the automorphism of $\mathbb{C}^{m} \times Y$ defined in (1.1). 
Proof. During this proof we will abbreviate $\omega_{\mathbb{C}^{m}}+\omega_{Y}=\omega_{P}$ and $g_{\mathbb{C}^{m}}+g_{Y}=g_{P}$.

By assumption $\zeta=\omega-\omega_{P}$ is exact. Let $\xi$ be any real 1 -form with $d \xi=\zeta$. Then $\bar{\partial} \xi^{0,1}=0$, so $\xi^{0,1}$ defines a class in $H^{0,1}\left(\mathbb{C}^{m} \times Y\right)$. By the Leray spectral sequence of the projection $\mathbb{C}^{m} \times Y \rightarrow \mathbb{C}^{m}$, there is an isomorphism $\Phi: H^{0,1}\left(\mathbb{C}^{m} \times Y\right) \rightarrow \mathcal{O}\left(\mathbb{C}^{m}, H^{0,1}(Y)\right)$ with $\Phi\left[\xi^{0,1}\right](z)=\left[\left.\xi^{0,1}\right|_{\{z\} \times Y}\right]$. This is made more explicit in the proof of [7, Lemma 4.1] if $\operatorname{dim}_{\mathbb{C}} Y=1$. The Beauville-Bogomolov-Calabi decomposition theorem [1, Thm 6.6] allows us to generalize the computations of [7] to our setting. Writing $\Phi\left[\xi^{0,1}\right]=f$ and identifying $H^{0,1}(Y)$ with the space of $g_{Y}$-parallel $(0,1)$-forms, $\left(\partial_{\bar{z}^{j}} f\right)(z)=0$ by [7, p.515], and similar arguments then show that $\left(\partial_{z^{j}} f\right)(z)$ is the $H^{0,1}$-class, or the parallel part with respect to $g_{Y}$, of the $(0,1)$-form $\left.\left(\partial_{z^{j}}\right\lrcorner \zeta\right)\left.\right|_{\{z\} \times Y}$ (independent of our choice of $\xi$ ). Since this form is uniformly bounded with respect to $g_{P}$, we learn that $f$ is a holomorphic polynomial of degree $\leqslant 1$. Changing $\xi$ by a suitable $g_{P}$-parallel form, we may assume that $f(0)=0$.

Writing $T_{\ell}^{*} \omega-\omega_{P}=T_{\ell}^{*}\left(\zeta+\tilde{\zeta}_{\ell}\right)$ with $\tilde{\zeta}_{\ell}=\omega_{P}-T_{-\ell}^{*} \omega_{P}$, and arguing as in the usual proof of the $i \partial \bar{\partial}$-lemma, one now easily checks that our proposition is equivalent to the following claim.

Claim. There exists a unique complex linear map $\ell: \mathbb{C}^{m} \rightarrow H^{0,1}(Y)$ such that there exists a real 1form $\tilde{\xi}$ such that $d \tilde{\xi}=\tilde{\zeta}_{\ell}$ and $\Phi\left[\tilde{\xi}^{0,1}\right]=-f$.

To prove uniqueness, notice that by the same reasoning as above, $\Phi\left[\tilde{\xi}^{0,1}\right]$ must be a holomorphic polynomial of degree $\leqslant 1$ with 1 -homogeneous part equal to $\ell$, so that necessarily $\ell=-f$. To prove that this choice of $\ell$ works, we first compute as in [8, p.382] that $\tilde{\zeta}_{\ell}=d \tilde{\xi}$ with

$$
\left.\tilde{\xi}=\ell^{\sharp}\right\lrcorner \int_{0}^{1} T_{-t \ell}^{*} \omega_{P} d t .
$$

Then it is easy to see that this choice of $\tilde{\xi}$ satisfies $\Phi\left[\tilde{\xi}^{0,1}\right]=\ell=-f$ as desired.

In the following proposition and in its proof, we fix $\omega_{\mathbb{C}^{m}}$ and $\omega_{Y}$ as reference metrics on $\mathbb{C}^{m}$ and $Y$. Also note that we will apply this proposition to $\zeta=\zeta_{t}=\omega_{t}-\left(\omega_{\mathbb{C}^{m}}+e^{-t} \omega_{Y}\right)$.

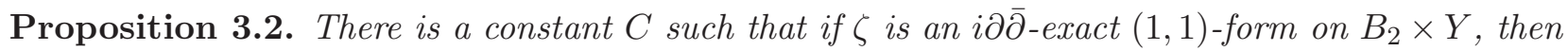
there is a function $\phi$ on $B_{2} \times Y$ with $i \partial \bar{\partial} \phi=\zeta$ and $\|\phi\|_{L^{\infty}\left(B_{1} \times Y\right)} \leqslant C\|\zeta\|_{L^{\infty}\left(B_{2} \times Y\right)}$.

Proof. By assumption there is a potential $\psi$ on $B_{2} \times Y$ such that $\zeta=i \partial \bar{\partial} \psi$. Let $\psi$ be the fiberwise average of $\psi$. This is a function on $B_{2}$. Writing $\underline{\zeta}=i \partial \bar{\partial} \underline{\psi}$, [15, (4.9)] shows that $\overline{\|} \underline{\zeta}\left\|_{L^{\infty}} \leqslant C\right\| \zeta \|_{L^{\infty}}$. The usual proof of the Poincaré lemma yields a $\overline{1}$-form $\underline{\xi}$ on $B_{2}$ with $d \underline{\xi}=\underline{\zeta}$ and $\|\bar{\xi}\|_{L^{\infty}} \leqslant C\|\underline{\zeta}\|_{L^{\infty}}$. Then $\bar{\partial} \underline{\xi}^{0,1}=0$, so $\bar{\partial}$-Neumann theory [5, Cor 8.10] produces a weak solution $\underline{\psi}^{\prime}$ to $\bar{\partial} \underline{\psi}^{\prime}=\underline{\xi}^{0,1}$ with $\left\|\underline{\psi}^{\prime}\right\|_{L^{2}} \leqslant C\left\|\underline{\xi}^{0,1}\right\|_{L^{2}}$. Defining $\underline{\psi}^{\prime \prime}=2 \operatorname{Im} \underline{\psi}^{\prime}$, it follows that $i \partial \bar{\partial} \underline{\psi}^{\prime \prime}=\underline{\zeta}$ as currents on $B_{2}$, hence in particular $\Delta \underline{\psi}^{\prime \prime}=\operatorname{tr}(\underline{\zeta})$ as distributions, $\overline{\text { so }} \underline{\psi}^{\prime \prime}$ is smooth with $\left\|\underline{\psi}^{\prime \prime}\right\|_{L^{\infty}\left(B_{1}\right)} \leqslant C\left(\left\|\underline{\psi}^{\prime \prime}\right\|_{L^{2}}+\|\underline{\zeta}\|_{L^{\infty}}\right)$. Thus, $\zeta=i \bar{\partial} \bar{\partial}\left(\psi-\underline{\psi}+\underline{\psi}^{\prime \prime}\right)$ with $\left\|\underline{\psi}^{\prime \prime}\right\|_{L^{\infty}\left(B_{1}\right)} \leqslant C\|\underline{\zeta}\|_{L^{\infty}}$. Then it remains to note that $\psi-\underline{\psi}$ has mean value zero on each fiber and $\bar{\Delta}_{Y}(\psi-\underline{\psi})=\operatorname{tr}\left(\left.\bar{\zeta}\right|_{Y}\right)$, so that $\|\psi-\underline{\psi}\|_{L^{\infty}} \leqslant C\|\zeta\|_{L^{\infty}}$ by fiberwise Moser iteration. Thus, a potential $\phi$ with the desired properties is given by $\phi=\psi-\underline{\psi}+\underline{\psi}^{\prime \prime}$.

3.2. Weak convergence of the functions $\sum_{j=1}^{m}\left|d z^{j}\right|_{\omega_{t_{i}}}^{2}$. Proposition 3.5 carries out Step 4. This is the first key difficulty of this paper. Let us first see why this step is nontrivial.

Example 3.3. Define a flat Kähler metric $\omega_{t}$ on $\mathbb{C}^{2}$ with coordinates $\left(w^{1}, w^{2}\right)=(z, y)$ by

$$
\omega_{t}=\frac{i}{2} g_{j \bar{k}} d w^{j} \wedge d \bar{w}^{k}, \quad\left(g_{j \bar{k}}\right)=\left(\begin{array}{cc}
1 & c e^{-t / 2} \\
c e^{-t / 2} & \left(c^{2}+1\right) e^{-t}
\end{array}\right),
$$

for a fixed $c \in \mathbb{R}$. This induces a flat Kähler metric on $\mathbb{C} \times Y$, where $Y=\mathbb{C} /(\mathbb{Z}+\mathbb{Z} i)$. Notice that the metric $\omega_{Y}$ in the sense of Theorem $\mathbf{A}(1)$ is given by $\omega_{Y}=\frac{i}{2}\left(c^{2}+1\right) d y \wedge d \bar{y}$. Then $\omega_{t}$ is uniformly bounded above and below by the product metric $\omega_{\mathbb{C}}+e^{-t} \omega_{Y}$ (where $\omega_{\mathbb{C}}=\frac{i}{2} d z \wedge d \bar{z}$ ), has the same determinant as this product metric up to a fixed constant factor, and converges to $\omega_{\mathbb{C}}$ with respect to any fixed norm as $t \rightarrow \infty$. But $\operatorname{tr}_{\omega_{t}}\left(\omega_{\mathbb{C}}\right)=\frac{1}{2}|d z|_{\omega_{t}}^{2}=c^{2}+1>m=1$ unless $c=0$. 
It is then clear that in order to prove that the limit of $\operatorname{tr}_{\omega_{t_{i}}}\left(\omega_{\mathbb{C}^{m}}\right)$ is $m$ in our situation, we need to find a way of proving that the "off-diagonal terms" as in (3.1) are negligible. The next proposition achieves this in a weak sense, using the fact that the difference of $\omega_{t}$ and $\omega_{\mathbb{C}^{m}}+e^{-t} \omega_{Y}$ is $i \partial \bar{\partial}$-exact (after Step 2,1). This fails to be true in Example 3.3 for $c \neq 0$. More precisely:

Example 3.4. In Example 3.3, the off-diagonal terms of $\omega_{t}-\left(\omega_{\mathbb{C}}+e^{-t} \omega_{Y}\right)$ are given by $c e^{-t / 2} \zeta$, where $\zeta=\frac{i}{2}(d z \wedge d \bar{y}+d y \wedge d \bar{z})$. On the universal cover, $\zeta=d \xi$ with $\xi=\operatorname{Im}(\bar{z} d y)$, and $\xi$ is invariant under the deck group. Also on the universal cover, $\zeta=i \partial \bar{\partial} \phi$ with $\phi=\operatorname{Re}(\bar{z} y)$, but there exists no pluriharmonic function $\pi$ on $\mathbb{C}^{2}$ such that $\phi+\pi$ is invariant under the deck group; indeed, if $\pi$ did exist, then $\phi+\pi$ would depend only on $z$ by Liouville's theorem applied fiberwise, contradicting the fact that $i \partial \bar{\partial}(\phi+\pi)=\zeta$, which contains $d y$ and $d \bar{y}$ components.

It is instructive to compare this with the formalism from [7] used in the proof of Proposition 3.1. With $\xi$ as above, we have that $\left.\xi^{0,1}\right|_{\{z\} \times Y}=\frac{i}{2} z d \bar{y}$, which is parallel on $\{z\} \times Y$, so the holomorphic function $f=\Phi\left[\xi^{0,1}\right]$ is given by $f(z)=\frac{i}{2} z d \bar{y}$. Moreover, if $T_{t}(z, y)=(z, y-A z)$ with $A=\frac{c}{c^{2}+1} e^{t / 2}$, then $\tilde{\omega}_{t}=T_{t}^{*} \omega_{t}$ has fundamental matrix $\operatorname{diag}\left(\frac{1}{c^{2}+1},\left(c^{2}+1\right) e^{-t}\right)$ and $\operatorname{tr}_{\tilde{\omega}_{t}}\left(\tilde{\omega}_{\infty}\right)=1$ as desired.

Proposition 3.5. For every test function $\eta \in C_{0}^{\infty}\left(\mathbb{C}^{m}\right)$ on the base,

$$
\lim _{i \rightarrow \infty} \int_{\mathbb{C}^{m} \times Y} \eta\left(\operatorname{tr}_{\omega_{t_{i}}}\left(\omega_{\mathbb{C}^{m}}\right)-m\right)\left(\omega_{\mathbb{C}^{m}}^{m} \wedge \omega_{Y}^{n}\right)=0 .
$$

Proof. We abbreviate $\omega_{\mathbb{C}^{m}}+e^{-t} \omega_{Y}=\omega_{P, t}$, so that $\omega_{t}=\omega_{P, t}+i \partial \bar{\partial} \phi_{t}$ by Steps 2 3 with $\phi_{t_{i}} \rightarrow 0$ in $C_{\text {loc }}^{1, \alpha}\left(\mathbb{C}^{m} \times Y\right)$ for a certain sequence $t_{i} \rightarrow \infty$, which is the sequence featuring in (3.2). For simplicity we will pretend that $\phi_{t} \rightarrow 0$ as $t \rightarrow \infty$. The following arguments are inspired by the method of proof of [15, Thm 4.1] (recall that we already used a variant of the latter in Step 2,3).

Thanks to the fact that $e^{n t} \omega_{t}^{m+n}=c\left(\omega_{\mathbb{C}^{m}}^{m} \wedge \omega_{Y}^{n}\right)$, it suffices to consider the expression

$$
\begin{aligned}
& {\left[\operatorname{tr}_{\omega_{t}}\left(\omega_{\mathbb{C}^{m}}\right)-m\right]\left(e^{n t} \omega_{t}^{m+n}\right)} \\
& =e^{n t}\left[(m+n) \omega_{\mathbb{C}^{m}} \wedge\left(\omega_{P, t}+i \partial \bar{\partial} \phi_{t}\right)^{m+n-1}-m\left(\omega_{P, t}+i \partial \bar{\partial} \phi_{t}\right)^{m+n}\right] \\
& =\sum_{k=0}^{m+n} e^{n t}\left[(m+n)\left(\begin{array}{c}
m+n-1 \\
k
\end{array}\right) \omega_{\mathbb{C}^{m}} \wedge \omega_{P, t}^{m+n-1-k}-m\left(\begin{array}{c}
m+n \\
k
\end{array}\right) \omega_{P, t}^{m+n-k}\right] \wedge\left(i \partial \bar{\partial} \phi_{t}\right)^{k} \\
& =\sum_{k=0}^{m+n}\left[\sum_{\ell=\max \{0, m-k\}}^{\min \{m, m+n-k\}} e^{(\ell-m+k) t} \frac{(m+n) !(\ell-m)}{k ! \ell !(m+n-k-\ell) !} \omega_{\mathbb{C}^{m}}^{\ell} \wedge \omega_{Y}^{m+n-k-\ell}\right] \wedge\left(i \partial \bar{\partial} \phi_{t}\right)^{k} .
\end{aligned}
$$

We now decompose $\phi_{t}=\underline{\phi_{t}}+\left(\phi_{t}-\underline{\phi_{t}}\right)$, where the underline denotes fiberwise averages, and further $i \partial \bar{\partial}\left(\phi_{t}-\underline{\phi_{t}}\right)=\alpha_{t}+\beta_{t}+\overline{\gamma_{t}}$ according to $\Lambda^{2}\left(\mathbb{C}^{m} \times Y\right)=\Lambda^{2} \mathbb{C}^{m} \oplus\left(\Lambda^{1} \mathbb{C}^{m} \otimes \Lambda^{1} Y\right) \oplus \Lambda^{2} Y$. Then

$$
\begin{aligned}
\left(i \partial \bar{\partial} \phi_{t}\right)^{k} & =\sum_{p=0}^{k}\left(\begin{array}{l}
k \\
p
\end{array}\right)\left(i \partial \bar{\partial} \underline{\phi_{t}}\right)^{p} \wedge\left(i \partial \bar{\partial}\left(\phi_{t}-\underline{\phi_{t}}\right)\right)^{k-p} \\
& =\sum_{p+q+r+s=k} \frac{k !}{p ! q ! r ! s !}\left(i \partial \bar{\partial} \underline{\phi_{t}}\right)^{p} \wedge \alpha_{t}^{q} \wedge \beta_{t}^{r} \wedge \gamma_{t}^{s} .
\end{aligned}
$$

Since $\left|i \partial \bar{\partial} \phi_{t}\right| \leqslant C$, the same holds for $i \partial \bar{\partial} \underline{\phi_{t}}$ by [15, (4.9)], and hence for $\alpha_{t}, \beta_{t}, \gamma_{t}$. However, because $\left.\omega_{t}\right|_{Y}=e^{-t} \omega_{Y}+\gamma_{t}$, we actually know that $\left|\gamma_{t}\right| \leqslant C e^{-t}$ with respect to any fixed background metric. Similarly, $\left|\beta_{t}\right| \leqslant C e^{-t / 2}$ by a Cauchy-Schwarz estimate with respect to $\omega_{t}$. As $2(\ell+p+q)+r=2 m$ for all nonzero terms in the big sum over $k, \ell, p, q, r, s$, we get $s+\frac{r}{2}=\ell-m+k$, so these bounds on $\left|\gamma_{t}\right|$ and $\left|\beta_{t}\right|$ compensate the $e^{(\ell-m+k) t}$ factors in (3.3), leading to an $O(1)$ overall bound.

Now even if $i \partial \bar{\partial} \phi_{t} \rightarrow 0$ in $L_{l o c}^{\mathbf{p}}$ for all $\mathbf{p}$ (which is more than we know from Step 3), we would not be able to prove that the terms with $p=q=0$ and $r>0$ are $o(1)$ rather than $O(1)$. Indeed, so far 
we have not made any real use of the fact that $\omega_{t}-\omega_{P, t}$ is $i \partial \bar{\partial}$-exact, and the corresponding terms in Example 3.3 are precisely the ones that obstruct the desired convergence. To fix this problem, we now multiply the whole expression by $\eta$ and integrate by parts, as follows.

- If $p<k$ in (3.4), throw one $i \partial \bar{\partial}$ from $\left(i \partial \bar{\partial}\left(\phi_{t}-\underline{\phi_{t}}\right)\right)^{k-p}$ onto $\eta$, then proceed as in (3.5) with $k$ replaced by $k-1$. In the resulting sum, $2(\ell+p+q+1)+r=2 m$ for all nonzero terms because of the additional factor of $i \partial \bar{\partial} \eta$, so again $s+\frac{r}{2}=\ell-m+k$ as before. Then it suffices to observe that $\left|\phi_{t}-\underline{\phi_{t}}\right|=O\left(e^{-t}\right)$ pointwise because $0 \leqslant n e^{-t}+\Delta_{Y}\left(\phi_{t}-\underline{\phi_{t}}\right)=\operatorname{tr}\left(\left.\omega_{t}\right|_{Y}\right) \leqslant C e^{-t}$.

- If $0<p=k$ in (3.4), then all nonzero terms in the remaining sum over $k, \ell$ satisfy $k+\ell=m$, so the $e^{(\ell-m+k) t}$ factor in $(\underline{3.3)})$ is bounded. Throw one $i \partial \bar{\partial}$ from $\left(i \partial \bar{\partial} \underline{\phi_{t}}\right)^{k}$ onto $\eta$ and observe that $\underline{\phi_{t}} \rightarrow 0$ locally uniformly on $\mathbb{C}^{m} \times Y$ because the same is true for $\phi_{t}$.

- If $0=p=k$ in (3.4), then we simply note that the $k=0$ term in (3.3) is zero anyway.

3.3. From weak to $L_{l o c}^{1}$ and pointwise. Proposition 3.6 below deals with Step 5 , 1 , proving that the weak convergence of Step 4 can be promoted to $L_{l o c}^{1}$ convergence. This is the second technical difficulty of this paper after Step 4. Proposition 3.8 then establishes Step 5,2, which is easier.

Proposition 3.6. Let $u_{i}$ be a sequence of smooth $\omega_{t_{i}}$-subharmonic functions on $\mathbb{C}^{m} \times Y$ such that $\left|u_{i}\right| \leqslant C$. Assume that there exists a constant function $u$ such that for all $\eta \in C_{0}^{\infty}\left(\mathbb{C}^{m}\right)$,

$$
\lim _{i \rightarrow \infty} \int_{\mathbb{C}^{m} \times Y} \eta\left(u_{i}-u\right)\left(\omega_{\mathbb{C}^{m}}^{m} \wedge \omega_{Y}^{n}\right)=0 .
$$

Then $u_{i}$ converges to $u$ in $L_{\text {loc }}^{1}$ with respect to the fixed volume form $\omega_{\mathbb{C}^{m}}^{m} \wedge \omega_{Y}^{n}$ on $\mathbb{C}^{m} \times Y$.

Proof. For subharmonic functions on $\mathbb{R}^{d}$ this is a special case of [10, Thm 3.2.12]. We will now adapt the proof of [10] to our setting. Because the $u_{i}$ are smooth and converge to a constant function $u$ as distributions, we can safely skip the part of the proof before (3.2.5) in [10].

The main point of the remainder of the proof in [10] is that if $v_{i}$ are smooth, uniformly bounded, subharmonic functions on $\mathbb{R}^{d}$ converging weakly to a smooth subharmonic function $v$ (not required to be constant), and if $\varphi_{\delta}$ is a standard family of radial mollifiers, then:

(1) $v_{i} \leqslant \varphi_{\delta} * v_{i}$ (this is the only point where the subharmonicity of $v_{i}$ is used),

(2) the sequence $\varphi_{\delta} * v_{i}$ is locally equibounded and equicontinuous for any fixed $\delta$,

(3) $\varphi_{\delta} * v_{i} \rightarrow \varphi_{\delta} * v$ in $L_{l o c}^{\infty}$ as $i \rightarrow \infty$, and $\varphi_{\delta} * v \rightarrow v$ in $L_{l o c}^{1}$ as $\delta \rightarrow 0$.

To recover these properties in our setting, we now replace Hörmander's smoothing operator $\varphi_{\delta} *$ by the time- $\delta^{2}$ heat evolution operator $H_{i, \delta} *$ associated with $\left(\mathbb{C}^{m} \times Y, \omega_{t_{i}}\right)$. Then:

$\left(1^{\prime}\right) u_{i} \leqslant H_{i, \delta} * u_{i}$,

$\left(2^{\prime}\right)\left|H_{i, \delta} * u_{i}\right| \leqslant C$ and $\sup _{\mathbb{C}^{m} \times Y}\left|\nabla^{\omega_{t_{i}}}\left(H_{i, \delta} * u_{i}\right)\right|_{\omega_{t_{i}}} \leqslant C \min \{\delta, 1\}^{-1}$, and

$\left(3^{\prime}\right)$ for all $\delta, R>0$ it holds that $\lim _{i \rightarrow \infty} \sup _{B_{R} \times Y}\left|\left(H_{i, \delta} * u_{i}\right)-u\right|=0$.

Let us prove these properties before showing that they imply the desired $L_{l o c}^{1}$ convergence. $\left(1^{\prime}\right)$ and $\left(2^{\prime}\right)$ immediately follow from our assumption that $\left|u_{i}\right| \leqslant C$, together with suitable versions of the maximum principle [12, Thm 15.2] and of the Cheng-Yau gradient estimate [11, Thm 3]. Here we rely on the fact that $\operatorname{Ric}\left(\omega_{t_{i}}\right) \geqslant 0$ to ensure that the constant $C$ of $\left(2^{\prime}\right)$ is uniform.

To prove $\left(3^{\prime}\right)$, fix $\delta, R>0$. Let $w_{i}=\left(H_{i, \delta} * u_{i}\right)-u$. By $\left(2^{\prime}\right)$ and Arzelà-Ascoli, some subsequence $w_{i_{j}}$ converges in $C^{0, \alpha}$ on $B_{R} \times Y$ to $w_{\infty}$, where $w_{\infty}$ is Lipschitz, and is constant on all fibers because $\left|w_{i}(z, y)-w_{i}\left(z, y^{\prime}\right)\right| \leqslant C_{\delta} e^{-t_{i} / 2} \operatorname{dist}_{\omega_{Y}}\left(y, y^{\prime}\right)$ by $\left(2^{\prime}\right)$. Thus, to prove that $w_{\infty}=0$, it suffices to prove that $\int \chi w_{i_{j}}\left(\omega_{\mathbb{C}^{m}}^{m} \wedge \omega_{Y}^{n}\right) \rightarrow 0$ for any fixed test function $\chi \in C_{0}^{\infty}\left(B_{R}\right)$ on the base, after passing to a further subsequence depending on $\chi$ if necessary 1 We will now prove this.

\footnotetext{
${ }^{1}$ As it turns out, we will not actually need to use the fact that $w_{\infty}$ and $\chi$ are pulled back from the base. However, the argument proving that $w_{\infty}$ is pulled back from the base also applies to $\eta_{\infty}$ below, and this will be crucial.
} 
The key step is to verify the identity

$$
\int_{\mathbb{C}^{m} \times Y} \chi w_{i_{j}}\left(\omega_{\mathbb{C}^{m}}^{m} \wedge \omega_{Y}^{n}\right)=\int_{\mathbb{C}^{m} \times Y} \eta_{i_{j}}\left(u_{i_{j}}-u\right)\left(\omega_{\mathbb{C}^{m}}^{m} \wedge \omega_{Y}^{n}\right), \eta_{i}=H_{i, \delta} * \chi .
$$

For this one uses the fact that $w_{i}=H_{i, \delta} *\left(u_{i}-u\right)$ because $u$ is constant, the Monge-Ampère equation $\omega_{t_{i}}^{m+n}=c e^{-n t_{i}}\left(\omega_{\mathbb{C}^{m}}^{m} \wedge \omega_{Y}^{n}\right)$ (here $c$ is some fixed normalizing factor), and standard Gaussian upper bounds for the heat kernel on a fixed manifold in order to justify applying Fubini's theorem.

The estimates $\left(2^{\prime}\right)$ hold verbatim for $\eta_{i}$ (with constants depending on $\chi$ ), so applying Arzelà-Ascoli once again, we learn that some subsequence $\eta_{i_{j_{k}}}$ converges in $C_{l o c}^{0, \alpha}$ on $\mathbb{C}^{m} \times Y$ to $\eta_{\infty}$, where $\eta_{\infty}$ is globally uniformly Lipschitz, and is constant on all fibers. In addition, by [12, Thm 13.4],

$$
\begin{aligned}
\left|\eta_{i}(x)\right| & \leqslant C \int_{\mathbb{C}^{m} \times Y}\left|B_{\omega_{t_{i}}}(x, \delta)\right|_{\omega_{t_{i}}}^{-1 / 2}\left|B_{\omega_{t_{i}}}\left(x^{\prime}, \delta\right)\right|_{\omega_{t_{i}}}^{-1 / 2} \exp \left(-\operatorname{dist}_{\omega_{t_{i}}}\left(x, x^{\prime}\right)^{2} / 5 \delta^{2}\right)\left|\chi\left(x^{\prime}\right)\right| \omega_{t_{i}}\left(x^{\prime}\right)^{m+n} \\
& \leqslant C_{\delta, R, \chi} \exp \left(-\operatorname{dist}_{\omega_{\mathbb{C}} m}\left(z, B_{R}\right)^{2} / C \delta^{2}\right), \quad x=(z, y) \in \mathbb{C}^{m} \times Y .
\end{aligned}
$$

Now fix any $\epsilon>0$, aiming to prove that for all $k \geqslant k_{\delta, R, \chi, \epsilon}$, the absolute value of the left-hand side of (3.7) for $j=j_{k}$ is bounded by some constant $C_{\delta, R, \chi}$ times $\epsilon$. To this end, decompose

$$
\eta_{i}=\chi_{S} \eta_{\infty, \beta}+\chi_{S}\left(\eta_{\infty}-\eta_{\infty, \beta}\right)+\chi_{S}\left(\eta_{i}-\eta_{\infty}\right)+\left(1-\chi_{S}\right) \eta_{i}
$$

where $\eta_{\infty, \beta}=\varphi_{\beta} * \eta_{\infty}$ for some standard radial mollifier $\varphi_{\beta}$ of radius $\beta$ on $\mathbb{C}^{m}$ (with $\beta=\beta_{S, \epsilon}$ to be determined) and where $\chi_{S}$ is some standard smoothing of the characteristic function of $B_{S}$ in $\mathbb{C}^{m}$ (with $S=S_{\delta, R, \epsilon}$ to be determined). Notice that $\left|\eta_{\infty}-\eta_{\infty, \beta}\right| \leqslant C_{\delta, \chi} \beta$ globally on $\mathbb{C}^{m}$ because $\eta_{\infty}$ is uniformly Lipschitz. Also, if $k \geqslant k_{\delta, \chi, S, \gamma}$, then $\left|\eta_{i_{j_{k}}}-\eta_{\infty}\right| \leqslant \gamma$ on $B_{2 S} \times Y$. Bringing these bounds and (3.8) into (3.9) for $i=i_{j_{k}}$, and using (3.7) for $j=j_{k}$, we learn that for all $k \geqslant k_{\delta, \chi, S, \gamma}$,

$$
\begin{aligned}
\left|\int_{B_{R} \times Y} \chi w_{i_{j_{k}}}\left(\omega_{\mathbb{C}^{m}}^{m} \wedge \omega_{Y}^{n}\right)\right| \leqslant & \left|\int_{\mathbb{C}^{m} \times Y} \chi_{S} \eta_{\infty, \beta}\left(u_{i_{j_{k}}}-u\right)\left(\omega_{\mathbb{C}^{m}}^{m} \wedge \omega_{Y}^{n}\right)\right| \\
& +C_{\delta, \chi} S^{2 m} \beta+C S^{2 m} \gamma+C_{\delta, R, \chi} \int_{S / 2}^{\infty} \exp \left(-\rho^{2} / C \delta^{2}\right) \rho^{2 m-1} d \rho
\end{aligned}
$$

provided that $S \geqslant 4 R$. Since $\chi_{S} \eta_{\infty, \beta} \in C_{0}^{\infty}\left(\mathbb{C}^{m}\right)$, our hypothesis (3.6) implies that the right-hand side of (3.10) is at most $\epsilon$ once $k \geqslant k_{\delta, R, \chi, S, \beta, \epsilon}$. Thus, it remains to choose $S=S_{\delta, R, \epsilon}$ large enough (roughly on the order of $|\log \epsilon|^{1 / 2}$ if $\delta$ and $R$ are given) and $\beta=\beta_{S, \epsilon}$ and $\gamma=\gamma_{S, \epsilon}$ small enough.

The upshot is that $\lim _{j \rightarrow \infty} \sup _{B_{R} \times Y}\left|\left(H_{i_{j}, \delta} * u_{i_{j}}\right)-u\right|=0$ for some sequence $i_{j}$ depending on $\delta, R$. Finally, we note that passing to such a subsequence is actually unnecessary because what we have really proved here is that given $\delta$ and $R$, every subsequence of $u_{i}$ has a further subsequence so that $\left(3^{\prime}\right)$ holds along this sub-subsequence; and this obviously implies $\left(3^{\prime}\right)$ as stated.

We are now in position to adapt the end of the proof of [10, Thm 3.2.12] to our setting. In fact, we can follow [10] almost word by word. Choose $\eta \geqslant 0$ in $C_{0}^{\infty}\left(\mathbb{C}^{m}\right)$ and $\epsilon>0$. By (3.6),

$$
\lim _{i \rightarrow \infty} \int_{\mathbb{C}^{m} \times Y} \eta\left(u-u_{i}+\epsilon\right)\left(\omega_{\mathbb{C}^{m}}^{m} \wedge \omega_{Y}^{n}\right)=\int_{\mathbb{C}^{m} \times Y} \epsilon \eta\left(\omega_{\mathbb{C}^{m}}^{m} \wedge \omega_{Y}^{n}\right) .
$$

Choose $\delta>0$, and choose $R>0$ with $\operatorname{supp}(\eta) \subset B_{R}$. Then $u_{i} \leqslant H_{i, \delta} * u_{i}$ by $\left(1^{\prime}\right)$, and $H_{i, \delta} * u_{i} \rightarrow u$ uniformly on $\operatorname{supp}(\eta) \times Y$ by $\left(3^{\prime}\right)$. Thus, $\eta\left(u-u_{i}+\epsilon\right) \geqslant 0$ for $i \geqslant i_{\eta, \epsilon}$. This implies that

$$
\limsup _{i \rightarrow \infty} \int_{\mathbb{C}^{m} \times Y} \eta\left|u_{i}-u\right|\left(\omega_{\mathbb{C}^{m}}^{m} \wedge \omega_{Y}^{n}\right) \leqslant 2 \int_{\mathbb{C}^{m} \times Y} \epsilon \eta\left(\omega_{\mathbb{C}^{m}}^{m} \wedge \omega_{Y}^{n}\right)
$$

by subtracting and adding $\epsilon$ on the left-hand side. The claim follows by letting $\epsilon \rightarrow 0$.

Remark 3.7. It seems likely that thanks to our assumption that $\omega_{t}$ is uniformly comparable to a collapsing product metric, the Gaussian upper heat kernel bounds used in (3.8) (and in the proof of Proposition [3.8) are true without any (Ricci) curvature conditions on $\omega_{t}$ or $\omega_{Y}$; see [6. Cor 15.25]. This seems less clear for the gradient estimates used in the proof of $\left(2^{\prime}\right)$ and $\left(3^{\prime}\right)$ above. 
Proposition 3.8. Let $v_{i}$ be a sequence of smooth $\omega_{t_{i}}$-subharmonic functions on $\mathbb{C}^{m} \times Y$ such that $v_{i}^{+} \leqslant C$. If $v_{i}^{+} \rightarrow 0$ in $L_{l o c}^{1}$ with respect to $\omega_{\mathbb{C}^{m}}^{m} \wedge \omega_{Y}^{n}$ on $\mathbb{C}^{m} \times Y$, then $\sup _{B_{1} \times Y} v_{i}^{+} \rightarrow 0$.

For clarity, let us note that this will be combined with Proposition 3.6 by setting $v_{i}=u_{i}-u$, so that $v_{i} \rightarrow 0$, hence $v_{i}^{+} \rightarrow 0$, in $L_{l o c}^{1}$. Here $u_{i}$ is one of the two sequences $\left|\partial_{z}\right|_{\omega_{t_{i}}}^{2}$ or $\sum_{j=1}^{m}\left|d z^{j}\right|_{\omega_{t_{i}}}^{2}$.

Proof. Let $H_{i, \delta}$ again denote the heat kernel at time $\delta^{2}$ associated with $\left(\mathbb{C}^{m} \times Y, \omega_{t_{i}}\right)$. As in the proof of Proposition 3.6, using the Karp-Li maximum principle [12, Thm 15.2] and the Li-Yau Gaussian upper heat kernel bounds [12, Thm 13.4], we obtain that

$$
\begin{aligned}
v_{i}(x) & \leqslant \int_{\mathbb{C}^{m} \times Y} H_{i, \delta}\left(x, x^{\prime}\right) v_{i}\left(x^{\prime}\right) \omega_{t_{i}}\left(x^{\prime}\right)^{m+n} \\
& \leqslant C \int_{\mathbb{C}^{m} \times Y}\left|B_{\omega_{t_{i}}}(x, \delta)\right|_{\omega_{t_{i}}}^{-1 / 2}\left|B_{\omega_{t_{i}}}\left(x^{\prime}, \delta\right)\right|_{\omega_{t_{i}}}^{-1 / 2} \exp \left(-\operatorname{dist}_{\omega_{t_{i}}}\left(x, x^{\prime}\right)^{2} / 5 \delta^{2}\right) v_{i}^{+}\left(x^{\prime}\right) \omega_{t_{i}}\left(x^{\prime}\right)^{m+n} .
\end{aligned}
$$

(The Li-Yau bounds produce a constant $C$ independent of $i$ because $\operatorname{Ric}\left(\omega_{t_{i}}\right) \geqslant 0$, but it seems very likely that this actually holds without any curvature conditions on $\omega_{t_{i}}$ or $\omega_{Y}$; see Remark 3.7.) We now assume that $\delta \leqslant 1$, aiming to make $\delta$ sufficiently small depending on $v_{i}$. Then Bishop-Gromov volume monotonicity yields that $\left|B_{\omega_{t_{i}}}(\hat{x}, \delta)\right|_{\omega_{t_{i}}} \geqslant C^{-1} e^{-n t_{i}} \delta^{2(m+n)}$ for $\hat{x}=x, x^{\prime}$. Moreover assume that $x \in B_{1} \times Y$, and decompose the domain $\mathbb{C}^{m} \times Y$ into $B_{2} \times Y$ and its complement. Using our assumption that $v_{i}^{+} \leqslant C$, it is then straightforward to deduce that

$$
\sup _{B_{1} \times Y} v_{i}^{+} \leqslant C \delta^{-2(m+n)} \int_{B_{2} \times Y} v_{i}^{+}\left(\omega_{\mathbb{C}^{m}}^{m} \wedge \omega_{Y}^{n}\right)+C \delta^{2-2(m+n)} \exp \left(-1 / C \delta^{2}\right) .
$$

Assuming that $\left\|v_{i}^{+}\right\|_{L^{1}\left(B_{2} \times Y\right)} \leqslant C^{-1}$, choose $\delta=\left|C \log \left\|v_{i}^{+}\right\|_{L^{1}\left(B_{2} \times Y\right)}\right|^{-1 / 2}$, where the $L^{1}$ norms are taken with respect to $\omega_{\mathbb{C}^{m}}^{m} \wedge \omega_{Y}^{n}$. It follows that $\sup _{B_{1} \times Y} v_{i}^{+} \rightarrow 0$ if $\left\|v_{i}^{+}\right\|_{L^{1}\left(B_{2} \times Y\right)} \rightarrow 0$.

3.4. Parallel vector fields imply splitting. The following proposition deals with Step 6 .

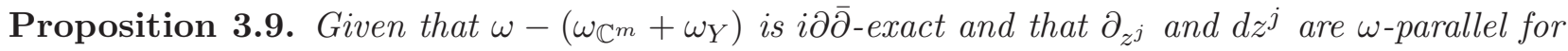
all $j \in\{1, \ldots, m\}$ with $\left|\partial_{z^{j}}\right|_{\omega}^{2}=\frac{1}{2}$ and $\left|d z^{j}\right|_{\omega}^{2}=2$, it follows that $\omega=\omega_{\mathbb{C}^{m}}+\omega_{Y}$.

Proof. Let $\tilde{\omega}$ be the pullback of $\omega$ to $\mathbb{C}^{m} \times \tilde{Y}$, where $\tilde{Y}$ is the universal cover of $Y$. Let $\tilde{P}$ be the flat de Rham factor of $\left(\mathbb{C}^{m} \times \tilde{Y}, \tilde{\omega}\right)$ spanned by the pullbacks of all $\omega$-parallel vector fields on $\mathbb{C}^{m} \times Y$. Then $\tilde{P}=\tilde{P}_{\mathbb{C}^{m}} \oplus \tilde{P}_{Y}$, where $\tilde{P}_{\mathbb{C}^{m}}$ is spanned by the pullbacks of $\partial_{z^{1}}, \ldots, \partial_{z^{m}}$ and $\tilde{P}_{Y}$ is the space of lifts of all $\omega_{Y}$-parallel vector fields on $Y$. (Here we have used the fact that $\nabla^{\omega}\left(d z^{j}\right)=0$ for all $j$, which implies that the fibers $\{z\} \times Y$ are $\omega$-totally geodesic and form an $\omega$-parallel family, and also that $\operatorname{Ric}\left(\left.\omega\right|_{\{z\} \times Y}\right)=0$, hence $\left.\omega\right|_{\{z\} \times Y}=\omega_{Y}$.) The main point to observe is that $\tilde{P}_{\mathbb{C}^{m}}$ is not a priori orthogonal to $\tilde{P}_{Y}$ with respect to the Euclidean metric $\left.\tilde{\omega}\right|_{\tilde{P}}$, but the orthogonal complement of $\tilde{P}_{Y}$ is the graph of a unique complex linear map $\ell^{\sharp}: \tilde{P}_{\mathbb{C}^{m}} \rightarrow \tilde{P}_{Y}$. Then the splitting $\tilde{P}=\tilde{P}_{\mathbb{C}^{m}} \oplus \tilde{P}_{Y}$ is orthogonal with respect to $T_{\ell}^{*} \tilde{\omega}$, and $T_{\ell}^{*} \omega=c_{\ell} \omega_{\mathbb{C}^{m}}+\omega_{Y}$ for some constant $c_{\ell}$. However, since $\omega$ is $i \partial \bar{\partial}$-cohomologous to $\omega_{\mathbb{C}^{m}}+\omega_{Y}$ by assumption, and $c_{\ell} \omega_{\mathbb{C}^{m}}$ is $i \partial \bar{\partial}$-cohomologous to $\omega_{\mathbb{C}^{m}}$, it follows from the uniqueness statement of Theorem $\mathrm{A}(2)$ that $\ell=0$.

This finishes Step [ and the proof of Theorem A. We conclude this paper with a remark on the automorphisms $T_{\ell}$. After reading the statement of Theorem $\AA(1)$ one may be tempted to guess that $\omega$ is always equal to a product Kähler form $S^{*} \omega_{\mathbb{C}^{m}}+\omega_{Y}$. We have proved that this is true if one replaces $\omega$ by $T_{\ell}^{*} \omega$ for a uniquely defined complex linear map $\ell: \mathbb{C}^{m} \rightarrow H^{0,1}(Y)$, where $T_{\ell}=$ id if and only if $\ell=0$. This still leaves the possibility that $\ell$ might always vanish. Now Examples 3.33 .4 show that $\ell \neq 0$ in general, and that this obstructs not only the conclusion that $\omega$ is a product form but also a key technical step of its proof (the statement of Proposition 3.5). Our final remark says that every nonzero linear map $\ell$ can be used to generate a counterexample in this way. 
Remark 3.10. Given a complex linear map $\ell: \mathbb{C}^{m} \rightarrow H^{0,1}(Y)$, the Kähler metric $T_{\ell}^{*}\left(\omega_{\mathbb{C}^{m}}+\omega_{Y}\right)$ is Ricci-flat with the same volume form as the product metric, and is parallel with respect to it. The vector fields $\partial_{z^{j}}$ are still parallel with respect to $T_{\ell}^{*}\left(\omega_{\mathbb{C}^{m}}+\omega_{Y}\right)$, but they do not define an isometric product splitting unless $\ell=0$; instead one needs to use the parallel vector fields $\left(\partial_{z^{j}},-\frac{\partial}{\partial z^{j}} \ell^{\sharp}\right)$.

\section{REFERENCES}

[1] W. Ballmann, Lectures on Kähler manifolds, European Mathematical Society, Zürich, 2006.

[2] L.A. Caffarelli, X. Cabré, Fully nonlinear elliptic equations, American Mathematical Society, Providence, RI, 1995.

[3] E. Calabi, Improper affine hyperspheres of convex type and a generalization of a theorem by K. Jörgens, Mich. Math. J. 5 (1958), 105-126.

[4] X.-X. Chen, Y. Wang, $C^{2, \alpha}$-estimate for Monge-Ampère equations with Hölder-continuous right hand side, Ann. Global Anal. Geom. 49 (2016), 195-204.

[5] J.-P. Demailly, $L^{2}$ estimates for the $\bar{\partial}$-operator on complex manifolds, lecture notes, Institut Fourier, Grenoble, 1996, see https://www-fourier.ujf-grenoble.fr/ demailly/books.html.

[6] A. Grigoryan, Heat kernel and analysis on manifolds, American Mathematical Society, Providence, RI, and International Press, Boston, MA, 2009.

[7] M. Gross, P.M.H. Wilson, Large complex structure limits of K3 surfaces, J. Differ. Geom. 55 (2000), 475-546.

[8] H.-J. Hein, Gravitational instantons from rational elliptic surfaces, J. Amer. Math. Soc. 25 (2012), 355-393.

[9] H.-J. Hein, V. Tosatti, in preparation.

[10] L. Hörmander, Notions of convexity, Birkhäuser, Boston, MA, 2007.

[11] B. Kotschwar, Hamilton's gradient estimate for the heat kernel on complete manifolds, Proc. Amer. Math. Soc. 135 (2007), 3013-3019.

[12] P. Li, Geometric analysis, Cambridge University Press, Cambridge, 2012.

[13] P. Li, L.-F. Tam, Linear growth harmonic functions on a complete manifold, J. Differ. Geom. 29 (1989), $421-425$.

[14] D. Riebesehl, F. Schulz, A priori estimates and a Liouville theorem for complex Monge-Ampère equations, Math. Z. 186 (1984), 57-66.

[15] V. Tosatti, Adiabatic limits of Ricci-flat Kähler metrics, J. Differ. Geom. 84 (2010), 427-453.

[16] V. Tosatti, B. Weinkove, X. Yang, The Kähler-Ricci flow, Ricci-flat metrics and collapsing limits, arXiv:1408.0161, to appear in Amer. J. Math.

[17] V. Tosatti, Y. Zhang, Infinite time singularities of the Kähler-Ricci flow, Geom. Topol. 19 (2015), 2925-2948.

[18] Y. Wang, Local regularity of the complex Monge-Ampère equation, Ph.D. thesis, Columbia University, 2013, see http://hdl.handle.net/10022/AC:P:20333

[19] S.-T. Yau, On the Ricci curvature of a compact Kähler manifold and the complex Monge-Ampère equation, I, Comm. Pure Appl. Math. 31 (1978), 339-411.

Department of Mathematics, Fordham University, Bronx, NY 10458, USA

E-mail address: hhein@fordham.edu 